\title{
Hierarchical ZSM-5 catalyst synthesized by a Triton X-100 assisted hydrothermal method
}

\author{
S. Narayanan ${ }^{\text {a,b }}$, J. Judith Vijaya a, ${ }^{\text {a, }}$, S. Sivasanker ${ }^{\mathrm{b}}$, Sihai Yang c, L. John Kennedy ${ }^{\mathrm{d}}$ \\ a Catalysis \& Nanomaterials Research Laboratory, Department of Chemistry, Loyola College (Autonomous), Chennai 600034, India \\ b National Centre for Catalysis Research, Indian Institute of Technology, Madras, Chennai 600 036, India \\ ' School of Chemistry, University of Nottingham, University Park, Nottingham NG7 2RD, United Kingdom \\ ${ }^{\mathrm{d}}$ Materials Division, School of Advanced Sciences, Vellore Institute of Technology (VIT) University, Chennai Campus, Chennai 600 127, India
}

\section{A R T I C L E I N F O}

\section{Article history:}

Received 11 April 2014

Accepted 17 June 2014

Published 20 November 2014

Keywords:

Heterogeneous catalysis

ZSM-5 zeolite

Hydrothermal synthesis

Selective oxidation

Surfactant

Triton X-100

\begin{abstract}
A B S T R A C T
ZSM-5 zeolite with a hexagonal cubic morphology was synthesized by a hydrothermal method using Triton X-100, a nonionic surfactant. The samples prepared with and without the surfactant were characterized by X-ray diffraction (XRD), Fourier transform infrared spectroscopy, $\mathrm{N}_{2}$ adsorption, high resolution transmission electron microscopy (TEM), high resolution scanning electron microscopy, energy dispersive X-ray analysis, and $\mathrm{NH}_{3}$ temperature-programmed desorption. The XRD patterns confirmed the formation of a pure ZSM- 5 crystalline phase without secondary phases. TEM images revealed that the hexagonal cubes were made of peanut-shaped nanoparticles with voids. The catalytic activity of the zeolite samples was evaluated using the selective oxidation of benzyl alcohol with tertiary-butyl hydrogen peroxide as the oxidant at $90^{\circ} \mathrm{C}$. The surfactant-assisted preparation yielded a zeolite that gave a higher conversion than the one prepared in the absence of the surfactant. The catalyst was retrieved and reused four times without significant loss in activity and selectivity.
\end{abstract}

(C) 2014, Dalian Institute of Chemical Physics, Chinese Academy of Sciences. Published by Elsevier B.V. All rights reserved.

\section{Introduction}

Zeolite molecular sieves are crystalline, three dimensional, and porous aluminosilicates. Zeolites have found numerous applications in petroleum refining and the chemical industry as heterogeneous catalysts, adsorbents, and ion exchangers due to their high surface area, uniform pore size, good thermal stability, and shape selective characteristics. Moreover, zeolite catalysts are environmentally friendly. The first zeolite mineral (stilbite) was discovered in nature by the Swedish mineralogist Cronstedt in 1756, and the first hydrothermal synthesis of a zeolite (levynite) was described by St. Clarie Deville in 1862. More than 170 zeolite framework structures are known, and many new structure types are reported each year. ZSM-5 (MFI type framework) is an important zeolite with pore diameters in the range of $0.54-0.56 \mathrm{~nm}$ and $\mathrm{Si} / \mathrm{Al}$ ratios that can be varied from 10 to 100 [1-5]. ZSM-5 was first synthesized by Argauer and Landolt in 1972 [6]. When their reactions are performed in the liquid phase, the small micropores in the zeolite limit the rate of diffusion of large molecules to the active catalytic sites, thereby limiting their applications in reactions involving large molecules. In order to overcome this problem, the synthesis of hierarchical zeolites containing both micro- and mesopores has drawn much attention $[7,8]$.

The synthesis of hierarchical zeolites using 'templating' and 'non-templating' approaches has been reported. The templating methods involve the use of (1) solid templates such as carbon materials of different structures, aerogels, polymers, res-

*Corresponding author. Tel: +91-44-28178200; Fax:+91-44-28175566; E-mail: jjvijayaloyola@yahoo.co.in, jjvijaya78@gmail.com 
ins, and biological templates and (2) supramolecular templates such as surfactants, and the use of indirect templating by the controlled deposition of zeolite crystals onto a template material. In the non-templating method (demetalation), a microporous zeolite is prepared first by hydrothermal synthesis in the presence or absence of a template. Then the synthesized microporous crystals are dealuminated (selective removal of $\mathrm{Al}$ from the framework) or desilicated (selective extraction of silicon from the framework) to leave a hierarchical pore structure. Dealumination is generally done by acid leaching or by steam treatment, and desilication is done by treatment in alkaline solutions [5,9-11]. In general, carbon black [12], colloid-imprinted carbon [13], carbon aerogels [14], biological templates [15], amphiphilic organosilane surfactants [16], tetrapropylammonium $\left(\mathrm{TPA}^{+}\right)$ions $[17,18]$, cationic polymers [19], and cetyltrimethylammonium bromide (CTAB) [20] have been used as the mesopore structure directing agent in the synthesis of hierarchical ZSM-5.

The selective oxidation of alcohols, particularly benzyl alcohol $(\mathrm{BzOH})$ to benzaldehyde $(\mathrm{BzH})$, is an important transformation in organic synthesis. Benzaldehyde has widespread applications in perfumery, and the dyestuff, pharmaceutical, and agro chemical industries [21]. The oxidation of alcohols has been extensively studied using stoichiometric inorganic oxidants like manganese and chromium salts, but these reagents are toxic and expensive and produce a large amount of hazardous waste [22]. Benzaldehyde is commercially produced either as a byproduct (low selectivity) in the oxidation of toluene or by the hydrolysis of benzyl chloride. The main drawback of the latter approach is that it results in the contamination of benzaldehyde with traces of chlorine, and this is therefore not suitable for perfumery and pharmaceutical applications [23-25]. The selective oxidation of benzyl alcohol in the liquid phase at lower reaction temperatures is preferred. Many homogeneous and heterogeneous catalysts have been reported for the liquid phase selective oxidation of benzyl alcohol with hydrogen peroxide [22,26,27]. Homogeneous catalysts exhibit better catalytic activity but are difficult to recover and reuse. Tert-butyl hydrogen peroxide (TBHP) is an organic peroxide and has many advantages in organic synthesis. It is a convenient source of active oxygen, safe to handle, and reasonably thermally stable [28]. ZSM-5 has not been reported for the selective oxidation of benzyl alcohol in the liquid phase with TBHP as the oxidant.

Here, we report the hydrothermal synthesis of ZSM-5 using Triton X-100 (Fig. 1) as the template. The textural properties of the samples were characterized by X-ray diffraction (XRD), Fourier transform infrared (FT-IR) spectroscopy, N2 adsorption, high resolution transmission electron microscopy (HR-TEM), high resolution scanning electron microscopy<smiles>CCOCCOCCOCCOc1ccc(C(C)(C)CC(C)(C)C)cc1</smiles>

Fig. 1. Structure of the nonionic surfactant Triton X-100.
(HR-SEM) and energy dispersive X-ray (EDX) analysis, and temperature-programmed desorption of ammonia ( $\mathrm{NH}_{3}-\mathrm{TPD}$ ). The ZSM-5 samples were tested for their catalytic activity in the liquid phase selective oxidation of benzyl alcohol to benzaldehyde with TBHP as the oxidant. The influences of reaction parameters on the conversion of the alcohol and selectivity to the aldehyde were investigated in detail.

\section{Experimental}

\subsection{Materials}

All the chemical reagents used in this work were analytical grade and were used without further purification. The synthesis of the zeolite was done using tetraethyl orthosilicate (TEOS, 98\%; Aldrich) as the silica source, aluminum isopropoxide (AIP; Aldrich) as the alumina source, tetrapropylammonium hydroxide (TPAOH, $25 \mathrm{wt} \%$; Aldrich) as the structure directing agent, Triton X-100 with the composition $\mathrm{C}_{14} \mathrm{H}_{22} \mathrm{O}\left(\mathrm{C}_{2} \mathrm{H}_{4} \mathrm{O}\right)_{n}(n=$ 9-10) (Sisco Research Laboratories Pvt. Ltd.) as the template, and deionized water.

\subsection{Synthesis of ZSM-5}

The detailed synthesis procedures are as follows. TPAOH (20 g) was dissolved in $33 \mathrm{~g}$ deionized water under stirring at room temperature for $1 \mathrm{~h}$, then $20.8 \mathrm{~g}$ of TEOS was added to the above solution, and the mixture was homogenized by stirring for $1 \mathrm{~h}$. Then, $0.727 \mathrm{~g}$ of AIP followed by $3.1 \mathrm{~g}$ of Triton X-100 was added dropwise into the above solution, and the mixture was sonicated at $35^{\circ} \mathrm{C}$ for $30 \mathrm{~min}$ in a sonicator bath for evenly dispersing AIP and Triton X-100 in the above solution. The mixture was allowed to react for $12 \mathrm{~h}$ at room temperature under magnetic stirring to ensure the complete hydrolysis of TEOS and AIP. The synthesis mixture was then aged at room temperature for $3 \mathrm{~h}$ without stirring. The gel composition of the resulting mixture was $56 \mathrm{SiO}_{2}: \mathrm{Al}_{2} \mathrm{O}_{3}: 14 \mathrm{TPAOH}: 3.83$ Triton $\mathrm{X}-100: 1500 \mathrm{H}_{2} \mathrm{O}$. The final homogeneous mixture was transferred into a Teflon-lined stainless steel autoclave. The crystallization reaction was carried out at $150{ }^{\circ} \mathrm{C}$ for $2 \mathrm{~d}$. The solid product was collected, centrifuged, filtered, then washed with deionized water, dried in an air oven, and then calcined at 550 ${ }^{\circ} \mathrm{C}$ for $5 \mathrm{~h}$ in a muffle furnace to remove the template. In the calcination of the final product, the temperature was raised from room temperature to $550{ }^{\circ} \mathrm{C}$ at a heating rate of $2{ }^{\circ} \mathrm{C} / \mathrm{min}$. The sample obtained using Triton X-100 as template was denoted as ZSM-5-WS.

For comparison, another ZSM-5 sample was synthesized using the same procedure but without Triton X-100. The gel composition of the resulting synthesis solution was 56 $\mathrm{SiO}_{2}: \mathrm{Al}_{2} \mathrm{O}_{3}: 14 \mathrm{TPAOH}: 1500 \mathrm{H}_{2} \mathrm{O}$. The sample synthesized without using Triton X-100 was represented as ZSM-5-WOS. The calculated $\mathrm{Si} / \mathrm{Al}$ ratio for both samples was 28. The flow chart for the synthesis of ZSM-5-WS and ZSM-5-WOS is illustrated in Fig. 2.

\subsection{Characterization}




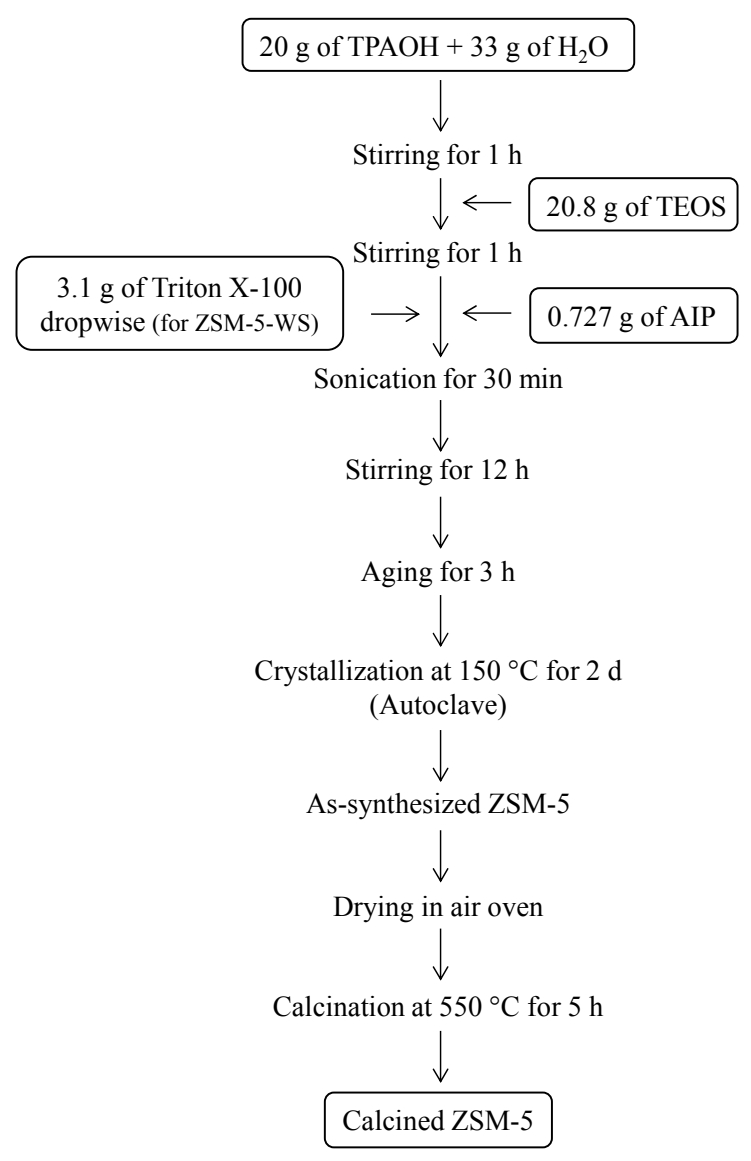

Fig. 2. Flow chart for the synthesis of ZSM-5-WS and ZSM-5-WOS.

The powder XRD patterns of the calcined zeolite samples were obtained using a Rigaku Miniflex II diffractometer with $\mathrm{Cu}$ $K_{\alpha}$ as the radiation source at a wavelength of $0.154 \mathrm{~nm}$. The patterns were obtained in the $2 \theta$ range of $5^{\circ}-60^{\circ}$ with $0.02^{\circ}$ step size. The FT-IR spectra were recorded using a Perkin Elmer FT-IR spectrophotometer in the wavenumber range of $4000-400 \mathrm{~cm}^{-1}$. The nitrogen adsorption isotherms at liquid $\mathrm{N}_{2}$ temperature were obtained using a Micromeritics instrument (ASAP 2020) for the determination of surface area and total pore volume. Prior to the adsorption measurements, the sample was degassed at $200{ }^{\circ} \mathrm{C}$ for $6 \mathrm{~h}$. HR-TEM measurement was carried out using a FEI TECNAI G ${ }^{2}$ model T-30 at an accelerating voltage of $250 \mathrm{kV}$ with electron diffraction (ED). The surface morphology and elemental composition of the sample were investigated using a Hitachi S-4800 SEM with an energy dispersive X-ray analyzer. $\mathrm{NH}_{3}$-TPD was conducted on a Micromeritics Chemisorb 2750 to measure the acid sites on the zeolite materials.

\subsection{Catalytic activity tests}

Liquid phase oxidation of benzyl alcohol was carried out in a batch reactor under atmospheric pressure. In a typical experiment, $0.15 \mathrm{~g}$ of the zeolite catalyst (ZSM-5-WS or ZSM-5-WOS), $20 \mathrm{mmol} \mathrm{BzOH}, 20 \mathrm{mmol}$ aqueous TBHP as the oxidant, and 10 $\mathrm{ml}$ ethyl acetate as solvent were placed in a round bottom flask equipped with a water condenser and thermometer. The re- sulting mixture was heated to $90{ }^{\circ} \mathrm{C}$ for $4 \mathrm{~h}$ and then cooled to room temperature. The oxidized products were separated by centrifugation. The products were analyzed by a gas chromatograph with a flame ionization detector using a SGE BPX70 capillary column (70\% Cyanopropyl Polysilphenylene-siloxane, $30 \mathrm{~m} \times 0.53 \mathrm{~mm} \times 0.50 \mu \mathrm{m}$ ) and $\mathrm{N}_{2}$ as a carrier gas.

\section{Results and discussion}

\subsection{Physicochemical characterization of the ZSM-5 samples}

Hierarchical zeolite nanoparticles have received increased attention because they exhibit properties that are significantly different from those of regular microporous materials that form large crystals. Therefore, the characterization of the hierarchical zeolites is important for understanding their catalytic properties.

\subsubsection{Structural properties}

Figure 3 shows the large angle XRD patterns of the ZSM-5 samples (ZSM-5-WS and ZSM-5-WOS). The patterns exhibit the characteristic diffraction peaks at $2 \theta=7.98^{\circ}, 8.82^{\circ}, 14.82^{\circ}$, $23.14^{\circ}, 23.96^{\circ}$, and $24.44^{\circ}$, which are indexed to the MFI topology (JCPDS no. 42-0024). The diffraction peaks are associated with the [011], [020], [031], [051], [303], and [313] planes with the $d$ spacing values of $1.11,1.00,0.59,0.39,0.37$, and $0.36 \mathrm{~nm}$, respectively. The very sharp reflections of the samples confirm the formation of a highly crystalline ZSM-5 phase, with no evidence of other crystalline phases. The peak intensity of the ZSM-WS zeolite was slightly lower than that of the ZSM-WOS zeolite, suggesting that the pore walls of the macropores were not completely converted into MFI structure. A lower degree of crystallization should also increase pore connectivity, which is advantageous for the diffusion of large molecules. The XRD result is similar to that reported in the literature [29].

The average crystallite size of the ZSM-5 zeolites was calculated using the Debye Scherrer formula [30], $L=0.89 \lambda /(\beta \cos \theta)$, where $L$ is the average crystallite size, $\lambda$ is the $\mathrm{X}$-ray wavelength $(0.154 \mathrm{~nm}), \theta$ is the Bragg diffraction angle, and $\beta$ is the full width at half maximum (FWHM) of the observed peak. The peak position and FWHM were obtained by fitting the peaks with two Gaussian curves in order to find the true peak posi-

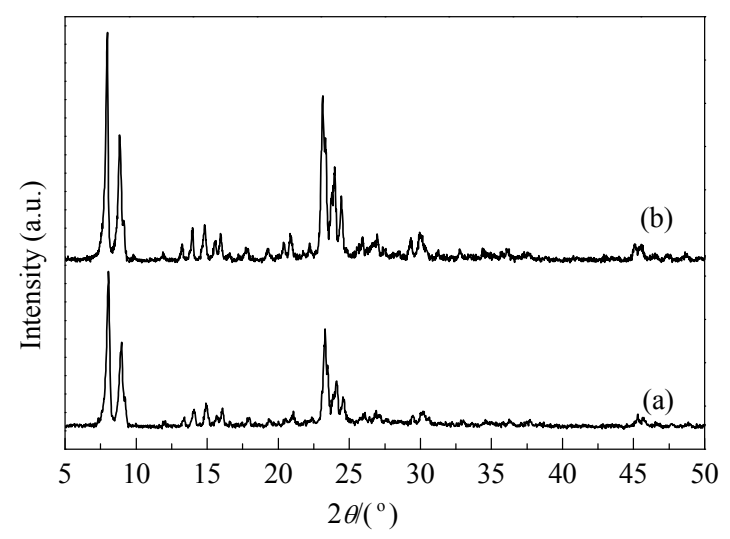

Fig. 3. XRD patterns of the samples. (a) ZSM-5-WS; (b) ZSM-5-WOS. 


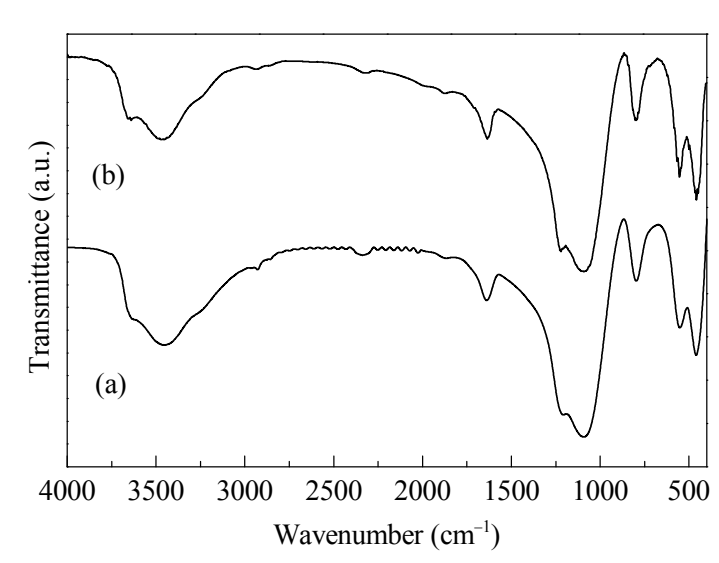

Fig. 4. FT-IR spectra of the samples. (a) ZSM-5-WS; (b) ZSM-5-WOS.

tion and width. The average crystallite sizes for ZSM-5-WS and ZSM-5-WOS calculated using the Scherrer formula were 15.5 and $30.1 \mathrm{~nm}$, respectively.

The formation of the ZSM-5 framework was further confirmed by the FT-IR spectra shown in Fig. 4. The FT-IR spectra reveal absorption bands at $1634,1225,1150-1050,796,549$, and $456 \mathrm{~cm}^{-1}$. The absorption bands at $1225 \mathrm{~cm}^{-1}$ (external asymmetric stretch), $1100 \mathrm{~cm}^{-1}$ (internal asymmetric stretch), $796 \mathrm{~cm}^{-1}$ (external symmetric stretch), and $456 \mathrm{~cm}^{-1}$ (T-0 bend) corresponded to siliceous materials. The framework vibration band at $549 \mathrm{~cm}^{-1}$, which has been assigned to the five-membered rings of MFI zeolites [31], can be used to determine the crystallinity of ZSM-5 zeolites [32]. Thus, both the XRD and FT-IR analysis reveal that the synthesized zeolite samples were the MFI type (ZSM-5).

\subsubsection{Textural properties}

Figure 5 shows the nitrogen adsorption isotherms of the ZSM-5-WS and ZSM-5-WOS. The samples gave type IV isotherms. Compared with ZSM-5-WOS, the ZSM-5-WS sample exhibited a broader hysteresis loop from $p / p_{0}=0.45$ to $p / p_{0}=$ 1 , which was due to the co-existence of both micropores and mesopores. The experimentally determined $\mathrm{Si} / \mathrm{Al}$ ratios, surface areas, and pore volumes of ZSM-5-WS and ZSM-5-WOS are listed in Table 1. The BET surface areas and pore volumes of

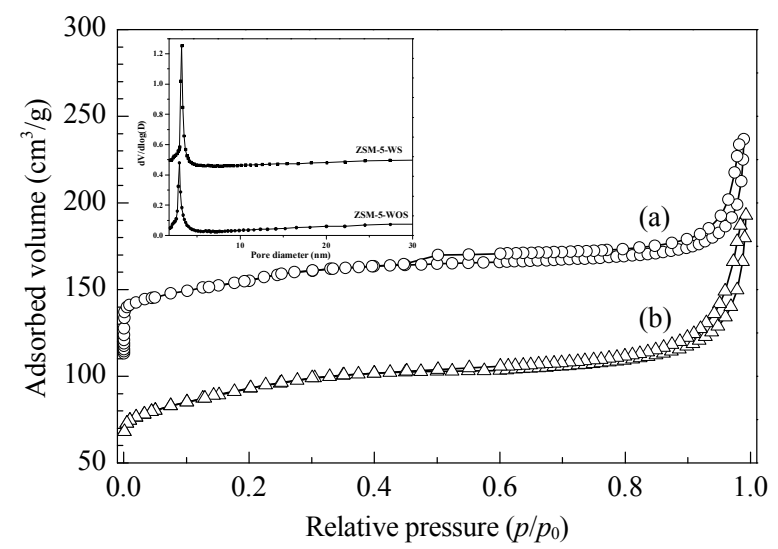

Fig. 5. $\mathrm{N}_{2}$ adsorption isotherms and pore size distribution (inset) of the zeolites. (a) ZSM-5-WS; (b) ZSM-5-WOS.
Table 1

Physicochemical properties of ZSM-5-WS and ZSM-5-WOS.

\begin{tabular}{lcccccc}
\hline Sample & Si/Al a & $\begin{array}{c}S_{\text {BET }} \\
\left(\mathrm{m}^{2} / \mathrm{g}\right)\end{array}$ & $\begin{array}{c}S_{\text {micro }} \text { b } \\
\left(\mathrm{m}^{2} / \mathrm{g}\right)\end{array}$ & $\begin{array}{c}V_{\text {total }} \\
\left(\mathrm{cm}^{3} / \mathrm{g}\right)\end{array}$ & $\begin{array}{c}V_{\text {micro }} \\
\left(\mathrm{cm}^{3} / \mathrm{g}\right)\end{array}$ & $\begin{array}{c}V_{\text {meso }} \mathrm{c} \\
\left(\mathrm{cm}^{3} / \mathrm{g}\right)\end{array}$ \\
\hline ZSM-5-WS & 27.2 & 345.2 & 123.9 & 0.369 & 0.092 & 0.276 \\
ZSM-5-WOS & 28.5 & 301.3 & 123.1 & 0.328 & 0.091 & 0.236 \\
\hline
\end{tabular}

${ }^{\text {a }}$ By XRF analysis. ${ }^{\mathrm{b}}$ Measured by the $t$-plot method. ${ }^{\mathrm{c}} V_{\text {meso }}=V_{\text {total }}-V_{\text {micro. }}$

ZSM-5-WS and ZSM-5-WOS were, respectively, $345.2 \mathrm{~m}^{2} / \mathrm{g}$ and $0.369 \mathrm{~cm}^{3} / \mathrm{g}$ (micropore volume, $0.092 \mathrm{~cm}^{3} / \mathrm{g}$ ) and $301.3 \mathrm{~m}^{2} / \mathrm{g}$ and $0.328 \mathrm{~cm}^{3} / \mathrm{g}$ (micropore volume, $0.091 \mathrm{~cm}^{3} / \mathrm{g}$ ). The mesopore volume $\left(0.276 \mathrm{~cm}^{3} / \mathrm{g}\right)$ of ZSM-5-WS is larger than that of ZSM-5-WOS $\left(0.236 \mathrm{~cm}^{3} / \mathrm{g}\right)$, although the micropore volumes of the two samples were nearly the same $\left(0.091 / 0.092 \mathrm{~cm}^{3} / \mathrm{g}\right)$. These results indicated that the additional mesoporosity in the ZSM-5-WS sample was generated by Triton X-100 added during the synthesis, which did not destroy the microporosity. The presence of a larger amount of mesoporosity in ZSM-5-WS should lead to faster diffusion of reactant molecules as compared to ZSM-5-WOS. The pore size distributions of ZSM-5-WS and ZSM-5-WOS are shown in the inset of Fig. 5. The mean pore diameter of ZSM-WS (3.9 $\mathrm{nm}$ ) was larger than that of ZSM-5-WOS (2.8 $\mathrm{nm})$, which indicates the formation of additional mesopores.

\subsubsection{Morphological characteristics}

Figure 6 presents the HR-TEM images and the particle size distribution of the ZSM-5-WS and ZSM-5-WOS samples. It was
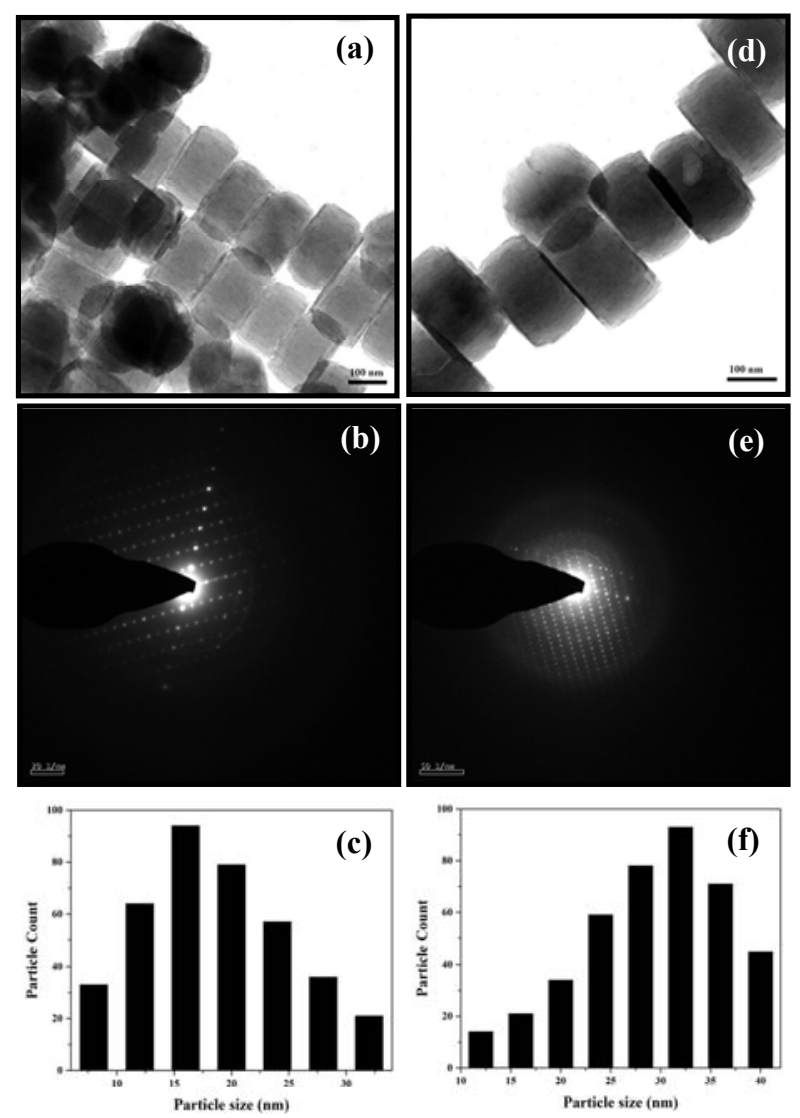

Fig. 6. HR-TEM images and particle size distribution of ZSM-5-WS (a,b,c) and ZSM-5-WOS (d,e,f). 
seen from the images that both the samples have disc-like nanostructures. Extremely small nanocrystallites of the zeolites were clearly seen in the pictures. A narrower particle size distribution was observed with ZSM-5-WS. The average sizes of the nanoparticles in the two samples were 12-20 nm and 24-32 nm for ZSM-5-WS and ZSM-5-WOS, respectively. The mean particle size determined by TEM was in good agreement with the average crystallite size calculated by the Debye-Scherrer formula from the XRD pattern. We concluded from the above results that Triton X-100 played a vital role in creating smaller particles with less agglomeration. The corresponding selected area electron diffraction (SAED) patterns of the zeolite samples are shown in Fig. 6(b) and (e). The patterns showed that the synthesized materials contained crystalline materials of a single crystalline phase. The SAED patterns gave the $d$ spacing values as $1.09,1.02,0.61,0.42,0.39$, and $0.35 \mathrm{~nm}$, which are consistent with those obtained from the XRD data.

Figure 7 shows the surface morphology of ZSM-5-WS and ZSM-5-WOS. The images revealed that the samples possess similar disc-like morphologies. However, the shape of ZSM-5-WOS was not uniform, while the shape of ZSM-5-WS was very regular. Figure 7(a) and (b) revealed that the sample ZSM-5-WS was made up of uniform hexagonal cubic crystals of 320-360 nm in length, which formed a disc-like morphology. In addition, it appeared that the individual crystals were actually aggregates made up of closely packed nanocrystals with sizes varying from 10 to $15 \mathrm{~nm}$. By comparison, in ZSM-5-WOS, the disc-like morphology was made up of hexagonal cubic crystals

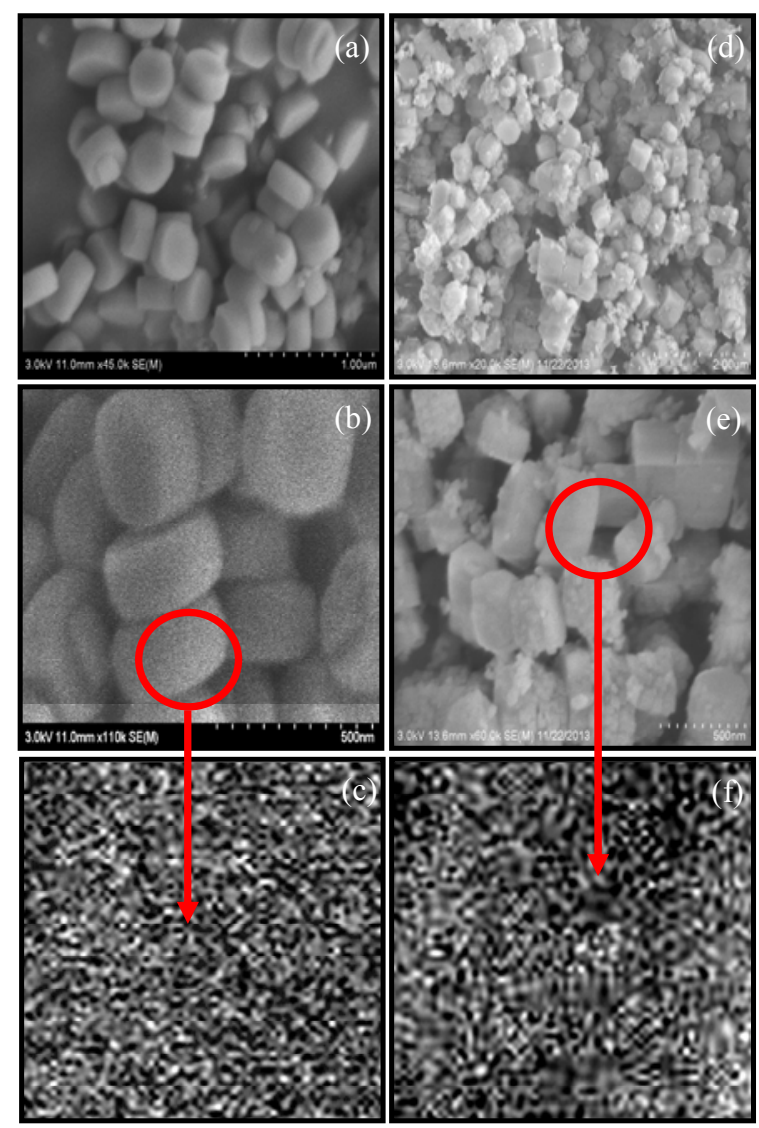

Fig. 7. HR-SEM images of ZSM-5-WS (a,b,c) and ZSM-5-WOS (d,e,f).

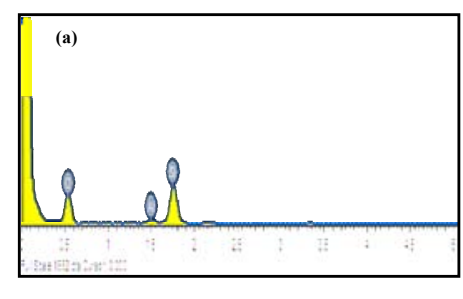

\begin{tabular}{|c|r|r|}
\hline Element & Wt \% & At \% \\
\hline O K & 55.19 & 68.31 \\
\hline Al K & 3.19 & 2.34 \\
\hline Si K & 41.62 & 29.34 \\
\hline Total & 100.00 & 99.99 \\
\hline
\end{tabular}
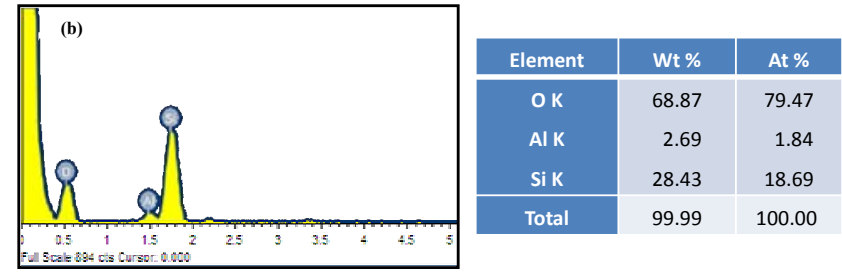

Fig. 8. EDX analysis of (a) ZSM-5-WS and (b) ZSM-5-WOS

of 300-400 nm in length (Fig. 7(d) and (e)). The surfaces of both the ZSM-5-WS and ZSM-5-WOS zeolite crystals were slightly rough. The rough surface may be due to the hexagonal cubic micro-blocks that were composed of numerous small nanosized primary ZSM-5 particles, which was suggested by the XRD results. In addition, Fig. 7(c) and (f) reveals the presence of nanosized primary ZSM-5 particles aggregated with voids.

There was only a small difference in the observed particle size measured by XRD, SEM, or TEM. The difference in the particle size of the ZSM-5-WS and ZSM-5-WOS samples was due to the fact that the SEM or TEM measurements were based on the difference between the visible grain boundaries, while the XRD calculations measured the extended crystalline region that diffracted the X-ray coherently. Hence, the XRD method has a more stringent criterion and gives smaller sizes [33].

The formation of a pure ZSM-5 zeolite phase was also confirmed by EDX analysis results presented in Fig. 8. The EDX results revealed that both ZSM-5-WS and ZSM-5-WOS were mainly composed of $\mathrm{Si}, \mathrm{Al}$, and $\mathrm{O}$. Hence, the results suggested the presence of a pure ZSM-5 phase without any impurity, in agreement with the XRD analysis.

\subsubsection{Acidity characterization results}

Acidic properties of the ZSM-5 catalysts were examined by $\mathrm{NH}_{3}$-TPD analysis. The TPD profiles of the ZSM-5-WS and ZSM-WOS catalysts are shown in Fig. 9. Generally, ZSM-5 zeo-

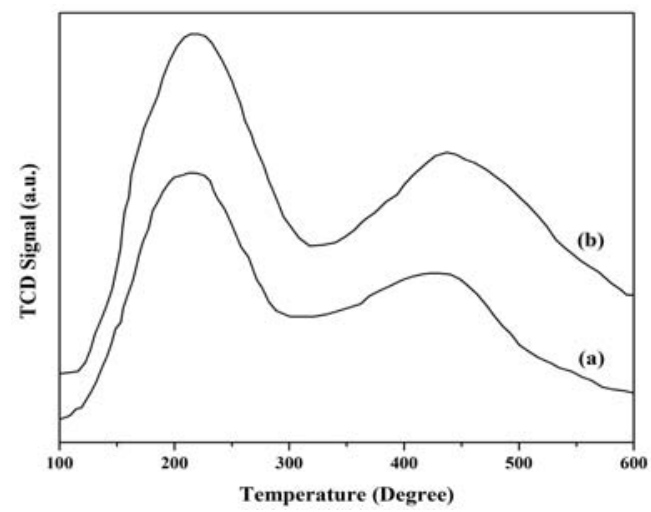

Fig. 9. $\mathrm{NH}_{3}$-TPD profiles of (a) ZSM-5-WS and (b) ZSM-5-WOS. 
lite has two desorption peaks at 200 and $400{ }^{\circ} \mathrm{C}$. The low temperature peak is due to weak acidic sites, while the high temperature peak is assigned to $\mathrm{NH}_{3}$ adsorbed on strong acid sites (Brönsted and Lewis acid sites) [34]. The $\mathrm{NH}_{3}$ desorption profile of ZSM-5-WS was similar to ZSM-5-WOS. For ZSM-WS, the high temperature desorption peak area was slightly lower than that of ZSM-5-WOS, indicating that the acidity of ZSM-5-WS was weaker than that of the latter, which was due to the presence of amorphous aluminosilicates on the macropore walls. The results are similar to the XRD analysis results.

\subsection{Catalytic activity in the selective oxidation of benzyl alcohol}

\subsubsection{Influence of catalyst amount}

$\mathrm{BzOH}$ conversion and selectivity to $\mathrm{BzH}$ as a function of catalyst amount are shown in Fig. 10. The conversion of $\mathrm{BzOH}$ increased with increasing amount of catalyst (ZSM-5-WS and ZSM-5-WOS) until the maximum conversions of $97.1 \%$ and $89.2 \%$ were achieved at a catalyst amount of $0.15 \mathrm{~g}$. No further increase in conversion was seen when $0.2 \mathrm{~g}$ catalyst was used. The lower conversion of $\mathrm{BzOH}$ with the lower loading of catalyst was due to fewer catalytic sites. The selectivity to $\mathrm{BzH}$, however, was found to decrease with increasing catalyst amount. This decrease in selectivity was because of the further oxidation of $\mathrm{BzH}$ to benzoic acid when more catalyst was present [35]. The conversion and selectivity remained nearly the same on further increasing the catalyst amount to $0.2 \mathrm{~g}$. This study showed that $0.15 \mathrm{~g}$ of the catalyst was sufficient for the reaction to reach optimum conversion and selectivity.

\subsubsection{Influence of reaction temperature}

The oxidation of $\mathrm{BzOH}$ over both ZSM-5-WS and ZSM-5-WOS was carried out at $60-100{ }^{\circ} \mathrm{C}$ while keeping the other reaction parameters the same. The results are illustrated in Fig. 11. The influence of reaction temperature on $\mathrm{BzOH}$ conversion is significant. The conversion of $\mathrm{BzOH}$ increased with

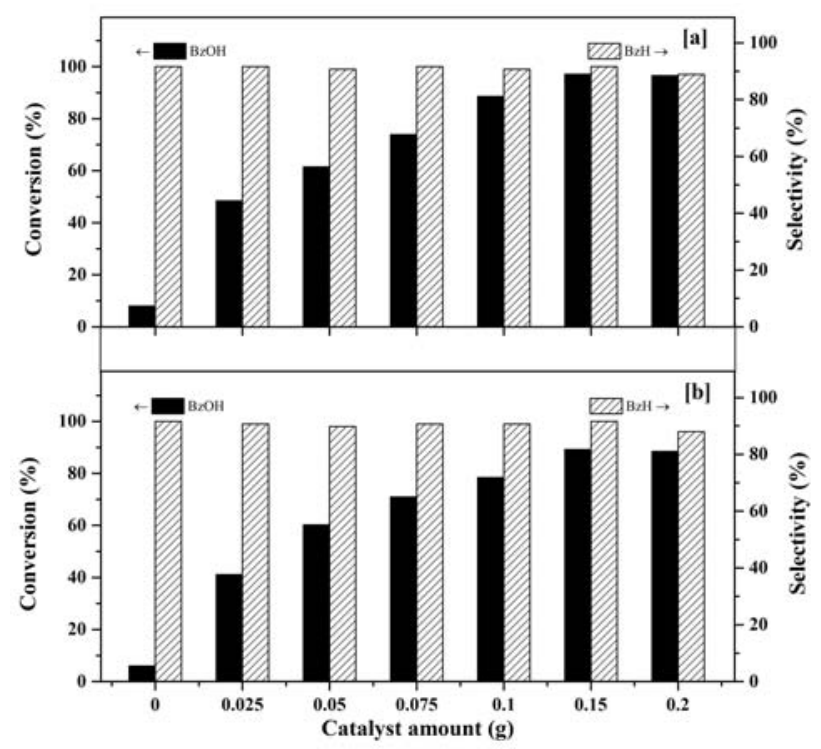

Fig. 10. Effect of catalyst amount on the oxidation of $\mathrm{BzOH}$. (a) ZSM-5-WS; (b) ZSM-5-WOS.

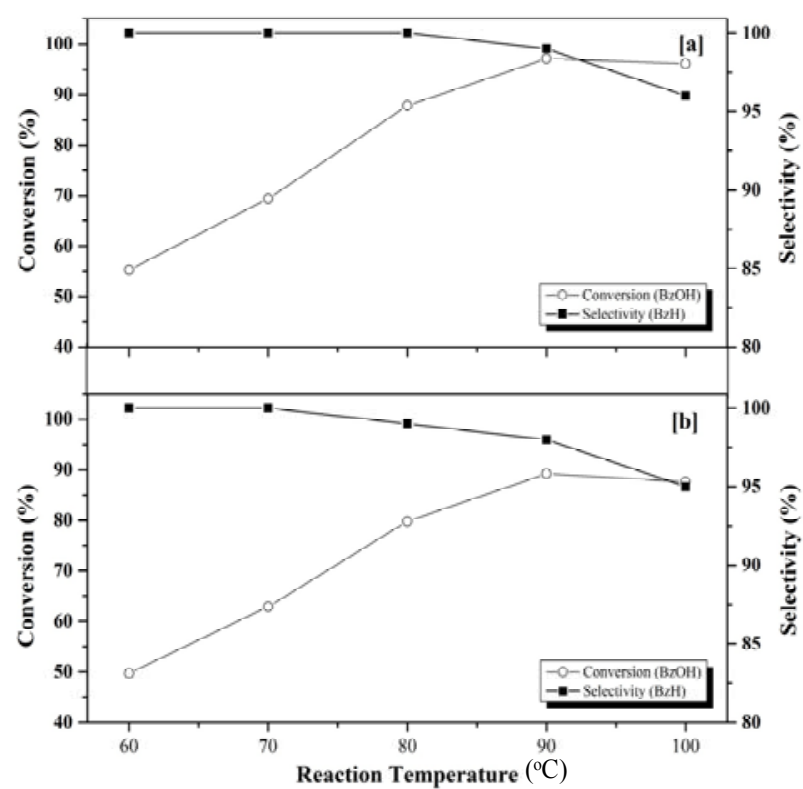

Fig. 11. Effect of reaction temperature on the oxidation of $\mathrm{BzOH}$. (a) ZSM-5-WS; (b) ZSM-5-WOS.

an increase in temperature from 60 to $90{ }^{\circ} \mathrm{C}$ from about $55 \%$ to $97 \%$ for ZSM-5-WS and from $50 \%$ to $89 \%$ for ZSM-5-WOS [36]. On further increasing the reaction temperature to $100{ }^{\circ} \mathrm{C}$, there was no change in the $\mathrm{BzOH}$ conversion, but the selectivity to $\mathrm{BzH}$ decreased. The conversion did not increase because of the decomposition of TBHP at the high temperature, and the selectivity to $\mathrm{BzH}$ decreased due to the over-oxidation of $\mathrm{BzH}$ to benzoic acid. Therefore, $90{ }^{\circ} \mathrm{C}$ was the optimum reaction temperature for the balance between $\mathrm{BzH}$ selectivity and $\mathrm{BzOH}$ conversion.

\subsubsection{Influence of solvent}

The solvent plays an important role in organic reactions. We investigated the influence of solvents with different polarities on the reaction. In the present study (Table 2), three different solvents, ethyl acetate, $n$-hexane, and toluene, were used to study their effect on the conversion of $\mathrm{BzOH}$ and selectivity to BzH. It is seen from Table 2 that the solvent has a remarkable effect on the oxidation reaction. Ethyl acetate was the best solvent. The BzOH conversion increased in the order ethyl acetate $>n$-hexane $>$ toluene. Although it is difficult to explain which property of the solvent influenced the reaction the most, the solvent should possess certain criteria. For example, it should dissolve both the substrate and the oxidant. The oxidant should be stable in the solvent. Low conversions were obtained with

Table 2

Effect of solvent on the selective oxidation of $\mathrm{BzOH}$.

\begin{tabular}{lccccc}
\hline \multirow{2}{*}{ Solvent } & \multicolumn{2}{c}{ ZSM-5-WS } & & \multicolumn{2}{c}{ ZSM-5-WOS } \\
\cline { 2 - 3 } \cline { 5 - 6 } & $\begin{array}{c}\text { Conversion } \\
(\%)\end{array}$ & \begin{tabular}{c} 
Selectivity \\
\cline { 5 - 6 }
\end{tabular} & & Conversion & Selectivity \\
Ethyl acetate & & & $(\%)$ & $(\%)$ \\
$n$-Hexane & 97.1 & 100.0 & & 89.2 & 100.0 \\
Toluene & 82.7 & 99.5 & & 75.4 & 98.7 \\
\hline
\end{tabular}


the low polarity solvent, which was $n$-hexane with a polarity index of 0.0 . This was attributed to the lower solubility of the reactant in the nonpolar solvent. In the case of toluene, although the polarity index is high (2.4), the activity was lower than that in $n$-hexane. This decrease was due to the lower solubility of TBHP in the aromatic solvent. Ethyl acetate being highly polar (polarity index of 4.4) with a large dielectric constant readily dissolved both TBHP and $\mathrm{BzOH}$. The higher solubility of the substrate and oxidant in ethyl acetate means that the reactants can easily approach the active sites of the catalyst.

In general, the conversion of $\mathrm{BzOH}$ increased with solvent polarity. Ethyl acetate was found to be the best solvent. As the oxidation of $\mathrm{BzOH}$ to $\mathrm{BzH}$ is a dehydrogenation reaction, the deprotonation of $\mathrm{BzOH}$ in an aprotic polar solvent, such as ethyl acetate, benefits the reaction. In addition, the relatively high solubility of $\mathrm{O}_{2}$ in ethyl acetate would also accelerate the oxidation [37]. As a consequence, ethyl acetate was the best solvent for the present catalytic system.

\subsubsection{Influence of the nature of the oxidant}

In order to design the best catalytic system, the effect of oxidants such as TBHP and $\mathrm{NaOCl}$ was examined (Table 3). In the absence of any oxidant, the reaction did not proceed. This clearly ruled out the possibility of the reaction occurring due to the participation of lattice oxygen alone. The selected oxidant should possess certain criteria. For example, it should dissolve in the solvent (ethyl acetate) and it should be stable. TBHP in ethyl acetate was found to be the most efficient oxidant to oxidize $\mathrm{BzOH}$. $\mathrm{NaOCl}$ is not an effective oxygen source for this catalytic system. TBHP readily mixes with $\mathrm{BzOH}$ and the solvent, and consequently the reactants can be adsorbed uniformly on the catalyst surface, therefore increasing conversion efficiency.

\subsubsection{Reusability of the catalyst}

The recycling of the catalyst is very important for industrial applications. Therefore, the reusability of the catalyst in the liquid phase oxidation of $\mathrm{BzOH}$ was evaluated. The results are presented in Table 4. After reaction, the catalyst was recovered by centrifugation and washed with ethanol, then dried $\left(110^{\circ} \mathrm{C}\right)$ and reused for another reaction cycle under the same condi-

Table 3

Effect of oxidant on the selective oxidation of $\mathrm{BzOH}$.

\begin{tabular}{lccccc}
\hline \multirow{2}{*}{ Oxidant } & \multicolumn{2}{c}{ ZSM-5-WS } & & \multicolumn{2}{c}{ ZSM-5-WOS } \\
\cline { 2 - 3 } \cline { 5 - 6 } & Conversion & Selectivity & & Conversion & Selectivity \\
& $(\%)$ & $(\%)$ & & $(\%)$ & $(\%)$ \\
\hline TBHP & 97.1 & 100.0 & & 89.2 & 100.0 \\
NaOCl & 79.4 & 98.7 & & 68.4 & 96.3 \\
\hline
\end{tabular}

Table 4

Reusability of ZSM-5 zeolite for the oxidation of BzOH with TBHP.

\begin{tabular}{|c|c|c|c|c|}
\hline \multirow[b]{2}{*}{ Run } & \multicolumn{2}{|c|}{ ZSM-5-WS } & \multicolumn{2}{|c|}{ ZSM-5-WOS } \\
\hline & $\begin{array}{c}\text { Conversion } \\
(\%)\end{array}$ & $\begin{array}{c}\text { Selectivity } \\
(\%)\end{array}$ & $\begin{array}{c}\text { Conversion } \\
(\%)\end{array}$ & $\begin{array}{c}\text { Selectivity } \\
(\%)\end{array}$ \\
\hline 1 & 97.1 & 100.0 & 89.2 & 100.0 \\
\hline 2 & 95.4 & 99.4 & 86.4 & 99.2 \\
\hline 3 & 96.5 & 98.7 & 88.5 & 98.2 \\
\hline 4 & 94.0 & 98.1 & 85.7 & 97.4 \\
\hline
\end{tabular}

tions. During the four runs investigated, the $\mathrm{BzOH}$ conversion decreased from $97.1 \%$ to $94.0 \%$ for ZSM-5-WS and from $89.2 \%$ to $85.7 \%$ for ZSM-5-WOS, revealing that there was only marginal decrease in conversion. The results indicated good reproducibility and stability for the two catalysts. It was also noticed that the selectivity to BzH decreased only slightly (from $100 \%$ to $98.1 \%$ for ZSM-5-WS and from $100 \%$ to $97.4 \%$ for ZSM-5-WOS). The marginal decrease in the performance of the catalysts with reuse was probably due to the blocking of the active sites by the formation of strongly adsorbed byproducts [22]. These results showed that ZSM-5 is a promising catalyst for the oxidation of $\mathrm{BzOH}$ with high activity and stability.

In this research, ZSM-5 zeolite nanoparticles prepared by the hydrothermal method were successfully used as an efficient and recyclable heterogeneous catalyst for the selective oxidation of $\mathrm{BzOH}$. We believe that our present work can lead to an alternative design methodology for the development of new efficient catalytic reaction systems for the synthesis of carbonyl compounds and other useful organic products.

\section{Conclusions}

Hierarchical ZSM-5 with a hexagonal cube morphology was successfully synthesized by a hydrothermal method using the nonionic surfactant Triton X-100 as template. Additional mesoporosity was created in the synthesis that used the Triton X-100 template. The ZSM-5 zeolite catalysts, ZSM-5-WS and ZSM-5-WOS, showed excellent activity in the selective oxidation of $\mathrm{BzOH}$ and gave high conversions of $97.1 \%$ and $89.2 \%$, respectively, when TBHP was the oxidant and ethyl acetate the solvent. The catalyst can be recovered and reused for a minimum of four runs without significant loss in conversion or selectivity. The results demonstrated that ZSM-5 zeolite nanoparticles are promising catalytic materials with high activity and stability for $\mathrm{BzOH}$ oxidation.

\section{Acknowledgments}

The authors are thankful to Prof. B. Viswanathan, NCCR, IIT-Madras for providing laboratory and characterization facilities to carry out this work. The authors would like to thank National Center for Nanoscience and Nanotechnology (NCNST), University of Madras for carrying out HRTEM studies.

\section{References}

[1] van Bekkum H, Flanigen E M, Jacobs P A, Jansen J C. Studies in Surface Science and Catalysis, Vol. 137, Introduction to Zeolite Science and Practice. Amsterdam: Elsevier, 2001

[2] Dyer A. An Introduction to Zeolite Molecular Sieves. New York: Wiley, 1988

[3] Corma A. Chem Rev, 1995, 95: 559

[4] Ozin G A, Kuperman A, Stein A. Angew Chem Int Ed, 1989, 28: 359

[5] Egeblad K, Christensen C H, Kustova M, Christensen C H. Chem Mater, 2008, 20: 946

[6] Argauer R J, Landolt G R. US Patent 3702886 A. 1972

[7] Groen J C, Peffer L A A, Moulijn J A, Pérez-Ramirez J. Microporous Mesoporous Mater, 2004, 69: 29 


\title{
Graphical Abstract
}

Chin. J. Catal., 2014, 35: 1892-1899 doi: 10.1016/S1872-2067(14)60177-7

\begin{abstract}
Hierarchical ZSM-5 catalyst synthesized by a Triton X-100 assisted hydrothermal method
S. Narayanan, J. Judith Vijaya*, S. Sivasanker, Sihai Yang, L. John Kennedy Loyola College (Autonomous), India; Indian Institute of Technology, India; University of Nottingham, United Kingdom; Vellore Institute of Technology (VIT) University, India
\end{abstract}
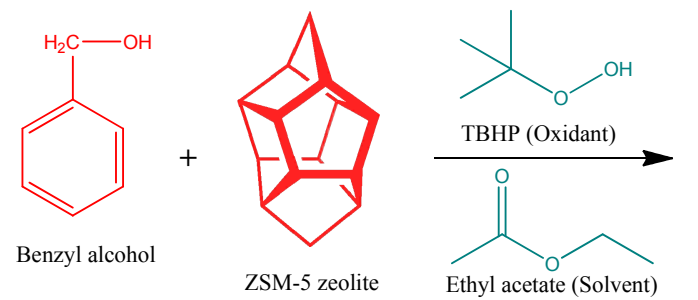<smiles>O=Cc1ccccc1</smiles>

Benzaldehyde

Hierarchical ZSM-5 was synthesized by a hydrothermal method using Triton X-100 as template, and it showed higher activity in the selective oxidation of benzyl alcohol in tert-butyl hydrogen peroxide (TBHP).

[8] Hartmann M. Angew Chem Int Ed, 2004, 43: 5880

[9] Lopez-Orozco S, Inayat A, Schwab A, Selvam T, Schwieger W. Adv Mater, 2011, 23: 2602

[10] Perez-Ramirez J, Christensen C H, Egeblad K, Christensen C H, Groen J C. Chem Soc Rev, 2008, 37: 2530

[11] Meng X J, Nawaz F, Xiao F S. Nano Today, 2009, 4: 292

[12] Koekkoek A J J, Xin H C, Yang Q H, Li C, Hensen E J M. Microporous Mesoporous Mater, 2011, 145: 172

[13] Kim S S, Shah J, Pinnavaia T J. Chem Mater, 2003, 15: 1664

[14] Tao Y S, Kanoh H, Hanzawa Y, Kaneko K. Colloids Surf A, 2004, 241: 75

[15] Valtchev V, Smaihi M, Faust A C, Vidal L. Stud Surf Sci Catal, 2004, 154: 588

[16] Choi M, Cho H S, Srivastava R, Venkatesan C, Choi D H, Ryoo R. Nat Mater, 2006, 5: 718

[17] Yang J H, Yu S X, Hu H Y, Zhang Y, Lu J M, Wang J Q, Yin D H. Chem Eng J, 2011, 166: 1083

[18] Petushkov A, Yoon S, Larsen S C. Microporous Mesoporous Mater, 2011, 137: 92

[19] Wang L F, Zhang Z, Yin C Y, Shan Z C, Xiao F S. Microporous Mesoporous Mater, 2010, 131: 58

[20] Chen G D, Jiang L, Wang L Z, Zhang J L. Microporous Mesoporous Mater, 2010, 134: 189

[21] Sharma R V, Soni K K, Dalai A K. Catal Commun, 2012, 29: 87

[22] Mallat T, Baiker A. Chem Rev, 2004, 104: 3037
[23] Pérez Y, Ballesteros R, Fajardo M, Sierra I, del Hierro I. J Mol Catal A, 2012, 352: 45

[24] Ajaikumar S, Pandurangan A. J Mol Catal A, 2008, 290: 35

[25] Yu Y Y, Lu B, Wang X G, Zhao J X, Wang X Z, Cai Q H. Chem Eng J, 2010, 162: 738

[26] Besson M, Gallezot P. Catal Today, 2000, 57: 127

[27] Dimitratos N, Lopez-Sanchez J A, Morgan D, Carley A, Prati L, Hutchings G J. Catal Today, 2007, 122: 317

[28] Bansal R K. Synthetic Approaches in Organic Chemistry. Burlington: Jones \& Bartlett Learing, 1996

[29] Wang L F, Yin C Y, Shan Z C, Liu S, Du Y C, Xiao F S. Colloids Surf A, 2009, 340: 126

[30] Ragupathi C, Vijaya J J, Narayanan S, Kennedy L J, Ramakrishna S. Chin J Catal (催化学报), 2013, 34: 1951

[31] Guo Y P, Wang H J, Guo Y J, Guo L H, Chu L F, Guo C X. Chem Eng J, 2011, 166: 391

[32] Fang Y M, Hu H Q.J Am Chem Soc, 2006, 128: 10636

[33] Bandyopadhyay S, Paul G K, Roy R, Sen S K, Sen S. Mater Chem Phys, 2002, 74: 83

[34] Ni Y M, Sun A M, Wu X L, Hai G L, Hu J L, Li T, Li G X. Microporous Mesoporous Mater, 2011, 143: 435

[35] Adam F, Ooi W T. Appl Catal A, 2012, 445-446: 252

[36] Chaudhari M P, Sawant S B. Chem Eng J, 2005, 106: 111

[37] Mondelli C, Ferri D, Grunwaldt J D, Krumeich F, Mangold S, Psaro R, Baiker A. J Catal, 2007, 252: 77 